\title{
BEAM VACUUM INTERCONNECTS FOR THE LHC COLD ARCS
}

\author{
$\underline{\text { R.Veness }}^{\circledR}$, J-C. Brunet, O.Grobner, P. Lepeule, C.Reymermier, G. Schneider, B. Skoczen, CERN \\ V.Kleimenov", I.Nikitine", IHEP Protvino
}

\begin{abstract}
Cold arcs of the LHC will consist of twin aperture dipole, quadrupole and corrector magnets in cryostats, operating at $1.9 \mathrm{~K}$. Beam vacuum chambers, along with all connecting elements require flexible 'interconnects' between adjacent cryostats to allow for thermal and mechanical offsets foreseen during machine operation and alignment. In addition, the beam vacuum chambers contain perforated beam screens to intercept beam induced heat loads at an intermediate temperature. These must also be connected with low impedance RF bridges in the interconnect zones.

The design of the beam vacuum interconnect is described in this paper. Features include a novel RF bridge design to maximise lateral flexibility during cryostat alignment and so-called 'nested' bellows to minimise the required length of the assembly.
\end{abstract}

\section{INTRODUCTION}

The Large Hadron Collider (LHC) is a $7 \mathrm{TeV}$ protonproton collider under construction at CERN, planned for start-up in 2005. Each of the 8 arcs of the machine will consist of repeating half-cells comprising three twin aperture dipole magnets, each $15.2 \mathrm{~m}$ long and a $6 \mathrm{~m}$ long 'short straight section' (SSS) containing lattice quadrupole and corrector magnets. Each arc is a continuous $2.5 \mathrm{~km}$ long cryostat operating at $1.9 \mathrm{~K}$.

All of the links between these magnets required for electrical power, cryogenics and beam vacuum (the subject of this article) must be connected at room temperature before the cryostat can be closed and cooled down. Thus, all these 'interconnects' must allow for the thermal contraction of the magnet cold masses. Due to the time and cost involved, the magnets will be warmed-up as seldom as possible. This means that the interconnects must have the flexibility to allow for re-alignments of the machine when cold or warm.

The beam vacuum system is described in detail elsewhere [1], however, a particular requirement for the LHC will be for a beam screen [2] inside the vacuum chambers to intercept beam induced heat loads at an intermediate temperature. These beam screens must also be connected with low impedance RF bridges between magnets.

\footnotetext{
${ }^{\circledR}$ Email: raymond.veness@ cern.ch

${ }^{\#}$ Scientific Associate at CERN
}

\section{DESIGN REQUIREMENTS}

Design offsets required for the beam vacuum interconnect can be divided into those expected during normal operation of the machine, transient offsets during machine cool-down and warm-up and 'exceptional' values due to possible faults, e.g. in the cryogenic system. The totals comprise mechanical tolerances, alignment tolerances and ground movements between alignment periods in addition to thermal expansion and contraction. For longitudinal offsets this sum is made arithmetically as the causes are principally thermal expansions. However, the lateral offsets are due to alignment and assembly tolerances, so the decision has been taken to assume a normal distribution of random errors and to calculate the offset for 3 standard deviations.

Following a review of similar RF bridge designs, in particular those for LEP [3], KEKB [4] and the SSC [5], a minimum contact force of $35 \mathrm{~g}$ per finger under all operation conditions was specified. An upper value for the contact force of $70 \mathrm{~g}$ was imposed on the sliding contact to avoid the possibility of particulate generation. Beam screens are cooled by supercritical helium flowing in two cooling tubes welded to each screen. Since the heat loads in the two beam apertures are not the same, the tubes must be swapped over between beam lines at each interconnect zone.

The envelope available for the interconnect is restricted in all directions. The length is limited to $480 \mathrm{~mm}$ between dipole magnets to minimise the loss of magnetic length around the machine. The radial space available for bellows and welding equipment is also limited by the other interconnect lines. In addition the beam screen must be passed through the $50 \mathrm{~mm}$ cold bore, which prevents the pre-mounting of equipment on one end.

The interconnects on either side of the SSS have additional requirements. On one side a pumping port is required on the beam lines. On the other side, the intermagnet zone contains a beam position monitor on each aperture.

\section{GENERAL DESCRIPTION}

The layout of a dipole-dipole beam vacuum interconnect is shown in figure 1 with the main features labelled. Each interconnect zone contains two such beam lines in 


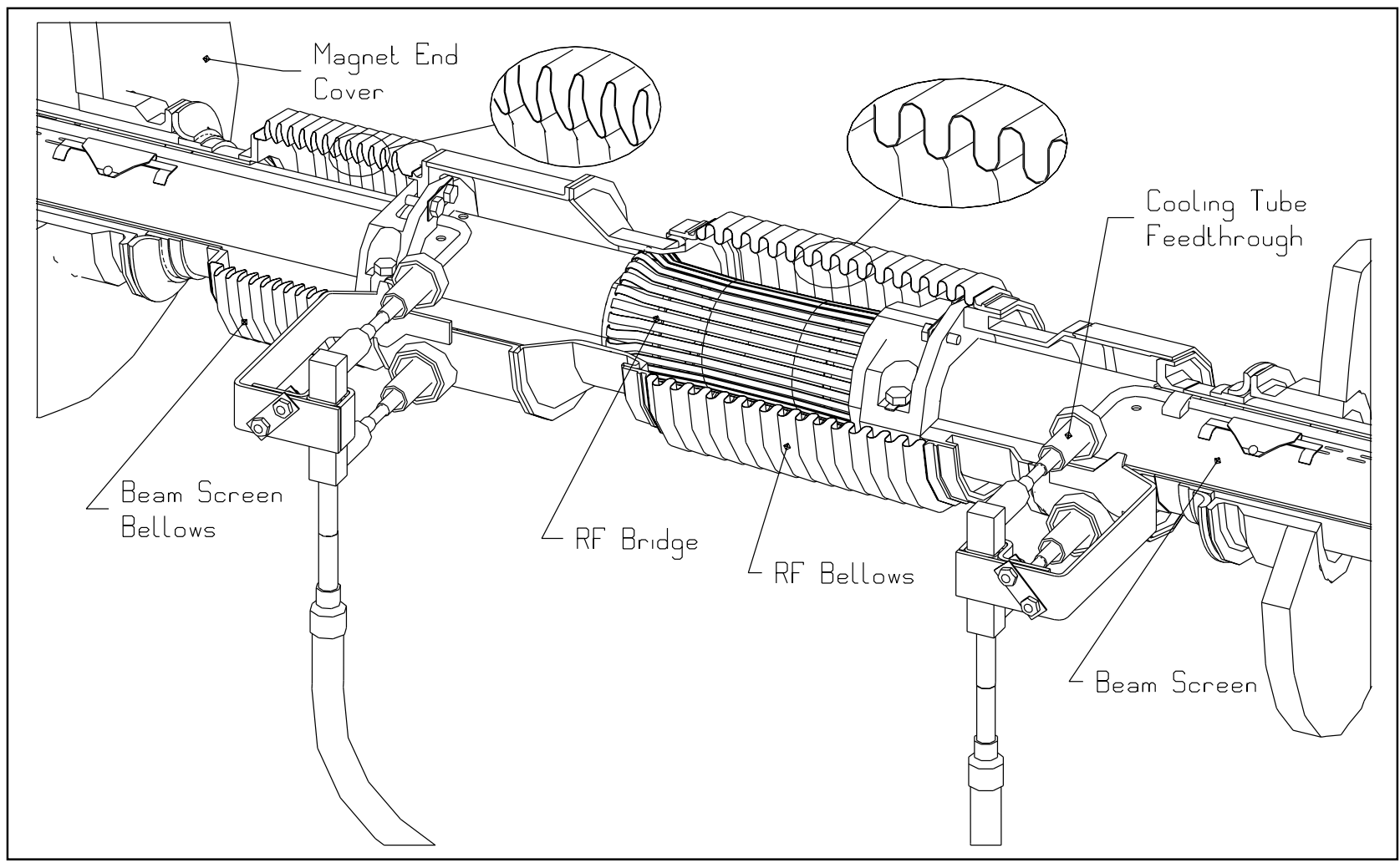

Figure 1: View of one Dipole-Dipole Beam Vacuum Interconnect.

parallel. A line contains two bellows; an 'RF bellows' around the RF bridge which allows for the thermal contraction and lateral offset of the magnet cold masses and a 'beam screen bellows' which absorbs differential thermal expansion between beam screen and magnet cold mass during thermal transients, preventing damage to the cooling tube feedthroughs. The required offsets of these elements both for normal machine operation (including thermal cycles) and for exceptional conditions (including re-alignment) are given in table 1 . The 'tolerances' column contains static mechanical and alignment values.

Table 1: Design Offsets of the Interconnect Flexible Elements in $\mathrm{mm}$

\begin{tabular}{|c|c|c|c|c|c|}
\hline & 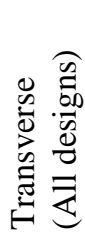 & 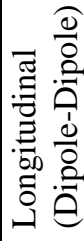 & 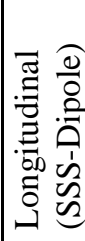 & 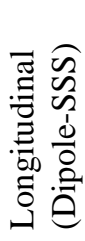 & 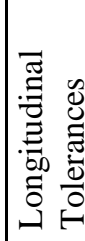 \\
\hline Beam Screen Bellows & & & & & \\
\hline Normal & 0 & 34 & 8 & 34 & 8 \\
\hline Exceptional & 0.5 & 75 & 29 & 75 & 8 \\
\hline RF-Bellows: Normal & 2 & 52 & 36 & 34 & 12 \\
\hline Exceptional & 4 & 62 & 38 & 57 & 12 \\
\hline RF-bridges: Normal & 2 & 52 & 36 & 34 & 12 \\
\hline Exceptional & 4 & 62 & 38 & 57 & 12 \\
\hline
\end{tabular}

Each line has four feedthroughs for the helium cooling tube cross-overs. There are fixed mechanical links between both ends of the beam screens and the interconnect to prevent stressing of these feedthroughs.

As explained in section 2, the SSS interconnects have some additional requirements. However, the same principals, and a majority of common components are used in all three designs.

Details of the other principal components are given in the following sections.

\section{RF BRIDGE DESIGN}

The RF bridge design concept was developed to avoid the high shear stress in contact fingers associated with the large lateral offsets required to re-align the machine when warm. The solution adopted was to provide a clearance, rather than a contact when warm and to use an change in the chamber wall diameter to apply the contact finger force only when the magnets are cold. This principle is shown in figure 2.

One RF bridge form was developed for all interconnects, fulfilling the 'normal' offset requirements of table 1 with a force per finger as defined in section 2. It was also designed so as not to plastify when under the 'exceptional' offsets from table 1.

Both beam screen ends have a transition from their standard 'racetrack' section to a circular section for the RF bridge. The profile of the fingers was optimised to minimise the variation of contact force over a range of offsets and to avoid buckling during magnet warm-up. 
The baseline material for the fingers is $0.2 \mathrm{~mm} \mathrm{CuBe} 2$ sheet which is electro-etched, formed and plated with 30 $\mu \mathrm{m}$ of silver. It is then rolled to shape and electron beam welded to a copper retaining ring.

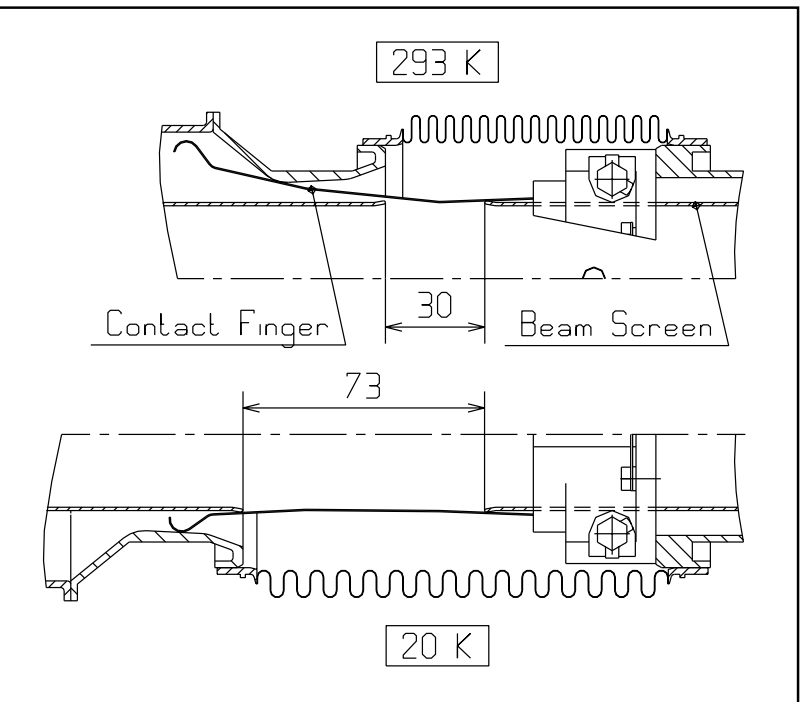

Figure 2: Section Through One Interconnect Line, Showing the RF bridge with the Beam Line at $293 \mathrm{~K}$ (Installed Position) and $20 \mathrm{~K}$ (Operating Position).

\section{BELLOWS DESIGN}

The minimised space in the interconnection zone imposes strong limitations on the length of both bellows units. The RF bellows is a hydroformed U-type profile, with offsets as described in table 1 . The size and axial stiffness were optimised for mechanical stability (buckling) and fatigue (thermo-mechanical cycles). The optimisation procedure is mainly based on the EJMA standards [6], extended to low temperature applications [7].

For the beam screen bellows a very compact 'nested bellows' has been designed for this application to compensate for the large transient axial offsets which occur during cool-down and warm-up (see table 1). The bellows profile, which has a large local curvature of the convolutions, has been analysed for local buckling, global mechanical stability and low cycle fatigue. Although this unit gives little lateral flexibility, it can accept 10-15\% more stroke for a given unit length than a U-type bellows. The alternative would be to use edge-welded bellows, however, this would imply more than $30 \mathrm{~km}$ of additional weld length in the $1.9 \mathrm{~K}$ system. A more detailed description of the bellows design is given in [8].

\section{MANUFACTURE AND ASSEMBLY}

The LHC will require a total of 3292 beam vacuum interconnect assemblies (1232 cryodipoles and 410 SSSs each with two apertures) which will be manufactured in European industry. All components except bellows and RF bridges will be manufactured in $316 \mathrm{LN}$ stainless steel. This grade has been chosen to ensure a fully austenitic structure at the operating temperatures, even after welding.

In order to maximise the reliability of the beam vacuum system, all sub-components will be manufactured without welds. Welds between components will be made by automated orbital TIG welding without the use of filler metal. The only exception will possibly be in the capillary exits, where laser welding is being considered to avoid the possibility of over-penetration into the capillaries containing high-pressure helium.

The design has been optimised to maximise the preassembly of the connections, either in a clean workshop or magnet assembly environment. Mechanical fixed points and RF bridges will be screwed in place inside the beam vacuum and held by tab washers. This leaves only the welds connecting the adjacent magnets, i.e. the RF bellows ends and cooling line cross-overs, to be performed in the tunnel. A number of intermediate quality controls and leak checks can therefore be performed to minimise the risk of interference to the very tight LHC installation schedule.

\section{WORK IN PROGRESS}

Manufacture of a pre-series of beam vacuum interconnects is under way. These will be installed in the LHC prototype string, consisting of 6 dipoles and 2 SSS's during 1999. This will allow a thorough testing of assembly and operation under cryogenic conditions. Extensive RF tests are under way to show that the bridge conforms to requirements.

\section{ACKNOWLEDGEMENTS}

The authors would like to thank F. Caspers, D. Chauville, G. Favre, H. Kos and F. Ruggiero for helpful discussions and their significant contributions to the design.

\section{REFERENCES}

[1] O.Grobner. The LHC Vacuum System. PAC 97, Vancouver, May 1997

[2] P.Cruikshank et.al. Mechanical Design of the LHC Beamscreen. PAC 97, Vancouver, May 1997.

[3] Specification for the Standard Bellows Assemblies of the LEP Main-Ring Vacuum system. CERN/I-1318/LEP. February 1985.

[4] K.Kanazawa, Y.Suetsugu. Development of a Bellows Assembly with RF-Shield for KEKB. EPAC 96, Sitges, June 1996.

[5] Design Report on the SSCL Prototype Synchrotron Radiation Liner System. SSCL note SSCL-SR-1224, September 1993.

[6] Standards of the Expansion Joint Manufacturers Association Inc., $6^{\text {th }}$ Edition 1993, Tarrytown, New York 10591. USA.

[7] B.Skoczen. Preliminary Optimum Design of the Expansion Joints for the LHC Dipole-Dipole Interconnects. CERN LHC Project Note 34, 1996

[8] B.Skoczen et.al. Development and Optimisation of Nested Bellows for the LHC Beam Line interconnection.CERN LHC/CRI Report 1-1998 